\title{
Is sugammadex alone sufficient to cause anaphylaxis?
}

\author{
Masaki Orihara $^{1} \cdot$ Tomonori Takazawa $^{2}$ (1) $\cdot$ Shigeru Saito ${ }^{1}$
}

Received: 13 December 2017 / Accepted: 8 January 2018 / Published online: 15 January 2018

(c) Japanese Society of Anesthesiologists 2018

To the editor:

We read with great interest and were very impressed by the report regarding sugammadex-induced anaphylaxis by Yamaoka et al. [1]. While the conclusion derived from the skin test results, which were positive for the sugammadex-rocuronium complex but negative for sugammadex alone, was correct, we believe that anaphylaxis after sugammadex administration does not necessarily result from the sugammadex-rocuronium complex acting as the antigen. The reason is because we have multiple cases in which sugammadex alone was sufficient to obtain positive test results both in vivo (i.e., skin tests) and in vitro (i.e., basophil activation tests), including a previously published case report [2]. Moreover, if the sugammadex-rocuronium complex expresses antigenicity after separate injections of rocuronium and sugammadex, anaphylaxis should have occurred in the intradermal tests that we have performed multiple times for our patient with sugammadex-induced anaphylaxis.

We would also like to discuss a technical issue associated with skin tests in this report. The maximum recommended concentration of rocuronium for intradermal tests is $50 \mu \mathrm{g} /$ $\mathrm{ml}$ (1:200) [3]. Although avoidance of false positive results is necessary, we do not believe that the excessive intradermal administration of rocuronium affected the outcome of this case.

Nevertheless, further research would be necessary to clarify the mechanism(s) of sugammadex-induced anaphylaxis.

\section{Compliance with ethical standards}

Conflict of interest The authors declare that they have no competing interests.

\section{References}

1. Yamaoka M, Deguchi M, Ninomiya K, Kurasako T, Matsumoto M. A suspected case of rocuronium-sugammadex complexinduced anaphylactic shock after cesarean section. J Anesth. 2017;31:148-51.

2. Takazawa T, Horiuchi T, Yoshida N, Yokohama A, Saito S. Flow cytometric investigation of sugammadex-induced anaphylaxis. $\mathrm{Br}$ J Anaesth. 2015;114:858-9.

3. Mertes PM, Malinovsky JM, Jouffroy L, Aberer W, Terreehorst I, Brockow K, Demoly P, Working Group of the SFAR and SFA, ENDA, EAACI Interest Group on Drug Allergy. Reducing the risk of anaphylaxis during anesthesia: 2011 updated guidelines for clinical practice. J Investig Allergol Clin Immunol. 2011;21:442-53.
This comment refers to the article available at https://doi. org/10.1007/s00540-016-2280-4.

Tomonori Takazawa

takazawt@gunma-u.ac.jp

1 Department of Anesthesiology, Gunma University Graduate School of Medicine, Maebashi, Gunma 371-8511, Japan

2 Intensive Care Unit, Gunma University Hospital, 3-39-15 Showa-machi, Maebashi, Gunma 371-8511, Japan 